\title{
Peer Group : A New Approach Of Nursing Intervention
}

\author{
${ }^{1,2}$ Suyanto Suyanto, ${ }^{3}$ Moses Glorino Rumambo Pandin Dr. M.Si., M.Phil., M.Psi., Psikolog \\ ${ }^{1}$ Program Doktor Nursing Faculty Universitas Airlangga Surabaya \\ ${ }^{2}$ Faculty of Nursing, Univesitas Islam Sultan Agung Semarang \\ ${ }^{3}$ Department of English Literature, Faculty of Humanities , Universitas Airlangga \\ Corespoden Author : Suyanto \\ Email : suyanto@unissula.ac.id
}

\begin{abstract}
Background: the development of nursing, especially related to the nursing intervention approach, is running so fast. This can be seen from the use of peer group support in nursing interventions in individual humans. The purpose of this literature is to find the impact of implementing nursing interventions using a peer group support approach.
\end{abstract}

Method: this literature review method uses JBI and Prisma on 120 articles taken from journal databases, namely Scopus, PubMed and Sciendirect.

Result: From the articles analyzed, it was found that the application of peer groups can improve individual abilities both in psychological and behavioral aspects.

Conclusion: the application of the peer group approach is able to be one of the approaches in the world of nursing in carrying out nursing actions today.

Keyword: Peer group support, peer group education and technology.

\section{INTRODUCTION}

One of the main components in the nursing paradigm is human. Humans are social creatures, where humans cannot live alone. Nurses with the role of care providers should re-realize the concept that humans are social creatures. In some cases where nurses have to provide intervention, it cannot be done only with an individual approach, but there is a need for a peer group support approach (1). One limitation of using an individual approach, especially in cases closely related to psychology, such as social isolation (2).

The limitations of the individual approach need other solutions that can be used by nurses in carrying out nursing actions. The peer group support approach can be one solution to these limitations. This is based on the fact that peer group support emphasizes togetherness among group members, especially seen from the same feelings towards what is being experienced (3).

The use of the peer group support approach can be used to improve individual behavior in the group. The peer group support approach can improve welfare, increase self-efficacy (3). Peer group education can also improve health, physical, emotional, and social well-being, reduce depression, and have a better future perspective (2). Various forms of peer group support are used, among others, the use of face to 
face peer groups, the use of technology, and the use of peer group education. The purpose of this literature review is to see the application of peer group support in improving aspects of psychology and patient behavior.

\section{METHODS}

The method used in this literature review uses the JBI and PRISMA protocol (4). Articles were obtained by writing keywords in 3 database journals, namely Scopus, Pubmed and Science Diret. The keywords included are peer group support, peer group education and technology peer group. The incluation criteria in this study are articles that appear selected from the year of publication, starting from 2019-2021, qualitative and quantitative research designs, and research areas in both hospitals and communities. Based on the PRISMA and JBI guidelines and inclusion criteria, 7 articles were obtained.

\section{RESULT}

The results of a review of 7 articles obtained from the journal database are as follows:

1. Effectiveness of Technologically Enhanced Peer Support in Improving Glycemic Management Among Predominantly African American, Low-Income Adults With Diabetes.

This study aims to determine the impact of the application of electronic health education combined with peer coaching. This study used a two armed randomized controlled trial. The intervention was given for 6 months. The inclusion criteria included patients with diabetes mellitus who were hospitalized, HbA1C $8.0 \%$ with age less than 70 years. The results of this study showed that there was no difference in the increase in the value of $\mathrm{HbA1C}$. The conclusion of this study is that there is no difference between the use of e health education technology compared to peer coaching(5).

2. Examining the effect of peer-support on self-stigma among persons living with HIV / AIDS

This study discusses differences in stigma ratings of HIV / AIDS sufferers between peer support groups. This study used a cross sectional study with the inclusion criteria of HIV / AIDS patients. The results showed that peer groups can reduce stigma in HIV / AIDS patients even though it is not enough to reduce stigma (chime). This study shows that although peer groups have not been able to significantly reduce the stigma of society, having a peer group can increase the confidence of HIV / AIDS patients with the support of peer groups of HIV / AIDS patients (6).

3. Peer-support: a coping strategy for nurses working at the Emergency Ambulance Service

The purpose of this study is about the experiences of nurses in using a peer supporting model. This research design is qualitative with the number of participants as many as 14 emergency room nurses. The results of this study need peer support in reducing emotional stress in the emergency room (7). This study is very interesting that nurses in the emergency room with work pressure that create emotional stress need peer support which is expected to reduce the emotional stress experienced by these nurses.

4. Perceived social support and quality of life among adolescents in residential youth care: a crosssectional study

Adolescents who have experienced family problems, behavior problems in residential institutions need social support in improving their quality of life. This study focuses on the relationship between social support and adolescent quality of life. The study design was cross sectional with 400 adolescents in 
residential institutions with an age range of more than $18-23$ years. The results show that there is a relationship between social support and adolescent quality of life. This study is important because adolescents who are in residential institutions need the importance of a lot of social support in their lives to improve their quality of life (8).

5. "Those People Motivate and Inspire Me to Take My Treatment." Peer Support for Adolescents Living With HIV in Cape Town, South Africa

This study examines the views of adolescents with HIV and caregivers regarding peer support. The study design was qualitative in 35 adolescents aged $12-19$ years who received treatment and 35 caregivers. The results of this study found that there are benefits to having peer group support, especially in improving communication between adolescents with HIV. A weakness of this study is the possibility of bias in peer group participants where they may not want to criticize their friends who are in the same group (9).

6. Mobile Peer-Support for Opioid Use Disorders: Refinement of an Innovative Machine Learning Tool

The use of information technology in implementing peer support needs to be developed. This study aims to create a peer group support-based platform for someone who experiences disorders due to drug use. 40 respondents were involved in this study. The results of this study are in the form of a platform which is the development of peer group support. In this research, we have not looked for the impact of this platform. This research is very good by combining information technology based on peer group support for someone who has a drug use disorder. In the future, there needs to be scientific evidence of the effectiveness of this platform (10).

7. The outcomes of peer-led diabetes education in comparison to education delivered by health professionals in Iranian patients

Patients with diabetes mellitus need to be educated. This study wants to compare the impact of education provided by health workers with peers on the self-care behavior of diabetes mellitus patients. The research design used clinical RCTs. A total of 40 respondents were included in this study, with incluation criteria consisting of age 40-65 years, people with type 2 diabetes, had a history of diabetes more than 6 months and were willing to participate in this study. The results of this study found that peer education can improve the self-care behavior of diabetes mellitus patients. The limitation of this study is that because the respondents were taken from the same hospital, it was possible that there was interaction between these respondents (11).

\section{DISCUSSION}

peer group is not an intervention, but peer group is an approach whose goal is to provide motivation, education and a community that supports each other (12). In its application, peer groups can be carried out into three approach activities, namely peer group support, peer group education and the use of peer group-based information technology. Peer group support is the most frequently used approach. The use of the peer group support approach tends to improve psychological and emotional aspects, although it cannot be separated from the behavioral aspects (3). Peer support can be done in the intensive care room where nurses can take this approach which is carried out on patients and families after leaving the incentive care room (13). Peer support can also improve the quality of life in patients with lupus. This happens because social support from peer groups can increase understanding of self-care management for lupus (1). The development of a peer group exercise model can also improve mental and physical 
health in cancer patients (14). The application of peer groups to mothers was also able to improve behavior to reduce obesity in their children (15). Various research results regarding peer group support can be used as a reference that peer group support can be used in various types of diseases and patient conditions.

Peer group education can be applied to adolescents, the elderly, diabetes patients, and nurses (16). Education using a peer group approach can improve self-care behavior in adolescents with asthma . For adolescents with peer led, they feel comfortable to have discussions with their peers. This is a factor that makes it easier for adolescents to apply self-care behavior (17). The application of peer led cancer with a peer support approach is also able to increase students' knowledge and self-esteem about cancer. This study used students' peers as group leaders in the cancer patient education process (18). Diabetes mellitus patients improve their quality of life with the peer group education approach (19)(20). Peer group education can be used in diabetes education, this is because the two methods are both able to increase patient understanding of blood sugar and lipid control (21).

Peer group-based technology applications have been developed just like virtual peer support for family can improve wellbeing of their family that have a chronic illness (22). One of them is the implementation of a peer support intervention program which is able to reduce the risk of postnatal depression (23). The use of peer group-based text massage can use short messages with an online approach where the results are that the use of online peer groups is more efficient (24). The use of social media with a peer group approach can provide emotional support and increase empowerment in diabetics (25).

\section{CONCLUSION}

peer group as an approach Nursing action needs to be done in both hospital and community services. Peer group support and peer group education, both conventional and technology-based, can be applied to patients with breast cancer, diabetes mellitus, HIV AIDS. This can have an impact on the increasingly varied approaches that can be taken by nurses in carrying out nursing actions.

\section{REFERENCE}

1. Williams EM, Egede L, Oates JC, Dismuke CL, Ramakrishnan V, Faith TD, et al. Peer approaches to self-management (PALS): Comparing a peer mentoring approach for disease self-management in African American women with lupus with a social support control: Study protocol for a randomized controlled trial. Trials. 2019;20(1):1-13.

2. Page-Reeves J, Murray-Krezan C, Regino L, Perez J, Bleecker M, Perez D, et al. A randomized control trial to test a peer support group approach for reducing social isolation and depression among female Mexican immigrants. BMC Public Health. 2021;21(1):1-18.

3. Toija AS, Kettunen TH, Leidenius MHK, Vainiola THK, Roine RPA. Effectiveness of peer support on health-related quality of life in recently diagnosed breast cancer patients: a randomized controlled trial. Support Care Cancer. 2019;27(1):123-30.

4. Kamioka H. Preferred reporting items for systematic review and meta-analysis protocols (prismap) 2015 statement. Japanese Pharmacol Ther. 2019;47(8):1177-85.

5. Shannalee R. Martinez, Maresha S. Gay and LZ. 乳鼠心肌提取 HHS Public Access. Physiol Behav. 
2016;176(1):139-48.

6. Chime $\mathrm{OH}$, Arinze-Onyia SU, Ossai EN. Examining the effect of peer-support on self-stigma among persons living with hiv/aids. Pan Afr Med J. 2019;34:1-10.

7. Carvello M, Zanotti F, Rubbi I, Bacchetti S, Artioli G, Bonacaro A. Peer-support: A coping strategy for nurses working at the emergency ambulance service. Acta Biomed. 2019;90(2):29-37.

8. Singstad MT, Wallander JL, Greger HK, Lydersen S, Kayed NS. Perceived social support and quality of life among adolescents in residential youth care: a cross-sectional study. Health Qual Life Outcomes [Internet]. 2021;19(1):1-12. Available from: https://doi.org/10.1186/s12955-02101676-1

9. Rencken CA, Harrison AD, Mtukushe B, Bergam S, Pather A, Sher R, et al. "Those People Motivate and Inspire Me to Take My Treatment." Peer Support for Adolescents Living With HIV in Cape Town, South Africa. J Int Assoc Provid AIDS Care. 2021;20:1-9.

10. Scherzer CR, Ranney ML, Jain S, Bommaraju SP, Patena J, Langdon K, et al. HHS Public Access. 2020;5(1):1-14.

11. Ahmadi Z, Sadeghi T, Loripoor M. The outcomes of peer-led diabetes education in comparison to education delivered by health professionals in Iranian patients. Health Educ Res. 2018;33(1):6472 .

12. Warshaw H, Edelman D. Building Bridges Through Collaboration and Consensus: Expanding Awareness and Use of Peer Support and Peer Support Communities Among People With Diabetes, Caregivers, and Health Care Providers. J Diabetes Sci Technol. 2019;13(2):206-12.

13. McPeake J, Hirshberg EL, Christie LM, Drumright K, Haines K, Hough CL, et al. Models of Peer Support to Remediate Post-Intensive Care Syndrome. Crit Care Med. 2019;47(1):e21-7.

14. Adlard KN, Jenkins DG, Salisbury CE, Bolam KA, Gomersall SR, Aitken JF, et al. Peer support for the maintenance of physical activity and health in cancer survivors: The PEER trial - A study protocol of a randomised controlled trial. BMC Cancer. 2019;19(1):1-15.

15. Cameron AJ, Charlton E, Walsh A, Hesketh K, Campbell K. The influence of the maternal peer group (partner, friends, mothers' group, family) on mothers' attitudes to obesity-related behaviours of their children. BMC Pediatr. 2019;19(1):1-8.

16. Backett-Milburn K, Wilson S. Understanding peer education: Insights from a process evaluation. Health Educ Res. 2000;15(1):85-96.

17. Richards et al. 乳鼠心肌提取 HHS Public Access. Physiol Behav. 2018;176(5):139-48.

18. Poudel K, Sumi N, Yano R. Impact of Peer-Led Cancer Education Program on Knowledge, Health Beliefs, Practice, and Self-Esteem Among Pairs of Nepalese High-School Students and Their Knowledge-Sharing Partners. Healthcare. 2021;9(1):64.

19. Ghasemi M, Hosseini H, Sabouhi F. Effect of peer group education on the quality of life of elderly individuals with diabetes: A randomized clinical trial. Iran J Nurs Midwifery Res. 2019;24(1):44-9.

20. Krishnamoorthy $Y$, Sakthivel M, Sarveswaran G, Eliyas SK. Effectiveness of peer led intervention in improvement of clinical outcomes among diabetes mellitus and hypertension patients $-A$ systematic review and meta-analysis. Prim Care Diabetes [Internet]. 2019;13(2):158-69. 
Available from: https://doi.org/10.1016/j.pcd.2018.11.007

21. Zhao X, Yu X, Zhang X. The Role of Peer Support Education Model in Management of Glucose and Lipid Levels in Patients with Type 2 Diabetes Mellitus in Chinese Adults. J Diabetes Res.

2019;2019.

22. Jeffrey A, Andracchio L, Dvorak M, Lomas P, Smith B, Borowitz D. Virtual Peer Support for People With Cystic Fibrosis and Their Family Members: A Program Evaluation. J Patient Exp. 2020;7(6):1748-54.

23. Shorey S, Chee C, Chong YS, Ng ED, Lau Y, Dennis CL. Evaluation of technology-based peer support intervention program for preventing postnatal depression: Protocol for a randomized controlled trial. J Med Internet Res. 2018;20(3).

24. Swendeman D, Arnold EM, Harris D, Fournier J, Comulada WS, Reback C, et al. Text-messaging, online peer support group, and coaching strategies to optimize the HIV prevention continuum for youth: Protocol for a randomized controlled trial. JMIR Res Protoc. 2019;8(8).

25. Gavrila V, Garrity A, Hirschfeld E, Edwards B, Lee JM. Peer Support Through a Diabetes Social Media Community. J Diabetes Sci Technol. 2019;13(3):493-7. 
